\title{
An Improved Novel Steganographic Technique For RGB And YCbCr Colorspace
}

\author{
Shweta Maurya, Vishal Shrivastava \\ M.Tech Scholar(C.S.E),Arya College Of Engineering And .T,Jaipur,Rajasthan \\ Professor(C.S.E), Arya College Of Engineering And I.T,Jaipur ,Rajasthan
}

\begin{abstract}
Steganography is the art of hiding the fact that communication is taking place, by hiding information in other information. Many different carrier file formats can be used, but digital images are the most popular because of their frequency on the Internet. For hiding secret information in images, there exists a large variety of steganographic techniques some are more complex than others and all of them have respective strong and weak points. Different applications have different requirements of the steganography technique used. For example, some applications may require absolute invisibility of the secret information, while others require a larger secret message to be hidden. This paper intends to give an overview of image steganography, its uses and techniques. It also attempts to identify the requirementsof a good steganographic algorithm and briefly reflects on which steganographic techniques are moresuitable for which applications.

The proposed work improve the imperceptibility of steganographicapproach and developed new algorithm using modifying approach. A comparative analysis of the proposed algorithm with the existing techniques using the statistical hypothesis testing framework is introduced in the proposed work and also we have evaluated the performance on comprehensive set of color images.
\end{abstract}

\subsection{Steganography Definition}

\section{Introduction}

Steganography is the art of hiding information imperceptibly in a cover medium. The word "Steganography" is of Greek origin and means "covered or hidden writing". As defined by [2], steganography is the art and science of communicating in such a way that the presence of a message can not be detected. The major goal of steganography is to increase communication security by inserting secret message into the digital image, modifying the redundancy or nonessential pixels of the image [1] , and has recently become important in a number of application areas especially military and intelligence agencies which require unobtrusive communications.Steganography includes a vast array of methods of secret communication that conceal the very existence of hidden information. Traditional methods include use of invisible inks, microdots etc. Modern day steganographic techniques try to exploit the digital media images, audio files, video files etc.

Steganography and cryptography are similar technique. Cryptography scrambles a message by using certain cryptographic algorithms for converting the secret data into unintelligible form. On the other hand, Steganography hides the message so that it cannot be seen. A message in cipher text might arouse suspicion on the part of the recipient while an "invisible" message created with steganographic methods will not. Anyone engaging in secret communication can always apply a cryptographic algorithm to the data before embedding it to achieve additional security. In any case, once the presence of hidden information is revealed, the purpose of steganography is defeated, even if the message content is not extracted or deciphered.

Another form of data hiding in digital images is watermarking. Digital watermarking is the process of embedding auxiliary information into a digital cover signal with the aim of providing authentication . A watermark is called robust with respect to a class of transformations if the embedded information can reliably be detected from the marked signal.

\section{Review Of Steganography}

The term steganography illustrates the art and science of hidden communication. By using steganography there is a chance to send messages so that nobody can detect the existence of the message. The message is embedded by weakening some characteristics of another media, which is called cover. Final output has equal properties to cover media, and also it includes our secret information. This new object is transmitted. If somebody is able to interpret this transmitted package, the secret message can be distinguished.While this transmitted package is really similar to cover media, detection of any embedded information is very difficult. For implementation of the steganography system,two algorithms are needed to be designed: one for hiding data and the other to extract this successfully. The main subject in embedding algorithm is to hide the secret message within the cover media without attracting any attention. The extraction algorithm has a simpler process and can be achieved by inversing the steps of embedding algorithm. 
The secret message usually is a text file or another image file which contains the secret information. This file is sent to the encoder unit in the first step. The encoder must be designed and implemented with high precision, to hide the secret message with a few distortion and changes in the cover image. Encoder unit usually needs a key to increase the security level of hiding method; this key is used in extraction phase too. Without using this key, the message willbe available without any impediment, if someone guesses theembedding or extraction algorithm.

Output of the encoder unit is called steganograme whichshould be close enough, to cover media. Then this image andthe key, which is used in embedding phase, are transmitted via a communication channel. In the next step this packageare applied to decoder unit. Output of the decoder unit isdelivered in the receiver side. The output of extraction unit isjust an estimate of secret message, because duringtransmition through the communication channel, thesteganograme is exposed to different types of noises, whichcan change the values of some bits.The application of steganographic technique can bebroadly classified as operating in two different domains, suchas spatial domain and frequency domain. In spatial domain,the embedding and hiding process are mostly carried out bybitwise manipulation. For example, manipulating the LSB inone of the color components in an image. While, thefrequency domain includes those which involve manipulationof transformed image such as Discrete CosineTransformation (DCT) and wavelet transformation. Suchmanipulation includes changing the value of the quantizedDCT coefficients.

\section{Proposed Approach}

The process of embedding data in this proposed algorithm is to RGB color cover image is taken up and its individual color channel are separated.Then change the colorspace to YCbCr.Select the $\mathrm{Cr} \mathrm{Channel}$ and divide it into $8 * 8$ size block. The text message to be embedded into cover image is encoded to form a bit sequence.

For each bit of the sequence are following method is apply.

(a)Take each block of $\mathrm{Cr}$ channel and then each block is transformed to the discrete cosine transformation domain.

(b)Select appropriate coefficient using the randomly generatred secret key known only to rightful owner.

(c)Insert each bit into these coefficient.

After placing the modified block into their corresponding position to form modified $\mathrm{Cr}$ channel,change back the colorspace from $\mathrm{YCbCr}$ to RGB.Thenstego image is formed. Therefore the perceptibility of image can be increased after changing the DCT values.

Perceptibility of this change is calculated with the Peak Signal-to-Noise Ratio (PSNR), which is used in the noisy communication channels to evaluate the ratio between the signal and the noise. The phrase PSNR is an engineering term for the ratio between the maximum possible power of a signal and the power of corrupting noise that affects the fidelity of its representation. It is most easily defined via the mean squared error (MSE) .

$$
M S E=\left(\frac{1}{M N}\right) \sum_{i=1}^{M} \sum_{j=1}^{N}\left(X i j-X^{\prime} i j\right)^{2}
$$

$$
\mathrm{PSNR}=10 . \log _{10} \mathrm{I}^{\wedge} 2 / \mathrm{MSE}
$$

where:

$X i j$ is the $i t h$ row and the $j t h$ column pixel in the original (cover) image,

$X i j$ is the $i t h$ row and the $j t h$ column pixel in the reconstructed (stego) image

Here, I is the maximum possible pixel value of the image. When the pixels are represented using 8 bits per sample, this is 255 . Usually the images with PSNR value less than 40, will be extremely ruined and can not be compared with the original image. For decoding the message in the receiver side, these parameters will be used to extract the secret message successfully. These parameters work as a key.Without any information about this key nobody can access the message correctly. There for the security level in this algorithm is higher than the JSteg algorithm which does not use any keys for communication.

\section{Results Of Algorithms}

Our colorization method produces believable colorized output images. We have colorized images in various color spaces and our method produced best results in YCbCrcolor space, we have presented a comparison between results obtained by our proposed method to 24 bit color image (improved LSB) [3][4] in Table (1-2). Theresults are compared with the original target image based on various parameters like Mean SquareError (MSE), Peak Signal to Noise Ratio (PSNR). As is apparent from this comparisonproposed method produces much better results.Images of various dimensions ranging from 256X256 to 1024x1024 were tested and the method proved Successful. 
Table 1: The PSNR(db) of Stego Images-Our First Steganography Method

\begin{tabular}{|l|c|}
\hline \multicolumn{1}{|l|}{ Technique } & Psnr \\
\hline [5] 24 Bit Color Image (Improved LSB) Method & 42.69 \\
\hline $\begin{array}{l}\text { [6]A Stegnograpy Implementation Based On DCT } \\
\text { Method }\end{array}$ & 40.67 \\
\hline \multicolumn{1}{|c|}{ Our Proposed method } & 50.99 \\
\hline
\end{tabular}

Table 2: Comparison of PSNR (in dB) of the stego image in different method

\begin{tabular}{|r|r|r|r|r|}
\hline Method/image & Lena & peppers & Babbon & Airplane \\
\hline Our Proposed method & 50.99 & 50.06 & 50.98 & 50.05 \\
\hline
\end{tabular}

\section{Conclusion}

Although only some of the main image steganographic techniques were discussed in this paper, one can see that there exists a large selection of approaches to hiding information in images. All the major image file formats have different methods of hiding messages, with different strong and weak points respectively. Where one technique lacks in payload capacity, the other lacks in robustness. For example, the patchwork approach has a very high level of robustness against most type of attacks, but can hide only a very small amount of information.Least significant bit (LSB) in both BMP and GIF makes up for this, but both approaches result in suspicious filesthat increase the probability of detection when in the presence of a warden. The image quality remains much conserved and robustness to the stego-attacks is also better in our proposed steganography technique based on colorspaces. This was the main concern of this research. The method presented has very good performance in imperceptibility.

\section{Reference-}

[1] Feng, J.B., Lin, I.C., Tsai, C.S., Chu, Y.P., 2006. Reversible watermarking: current status and key issues. International Journal of Network Security 2 (May), 161-170.

[2] C. Cachin, "An Information-Theoretic Model for Steganography”, Proceedings of 2nd Workshops on Information Hiding, MIT Laboratory for Computer Science, May 1998

[3] Smriti Kumar, AyushSwarnkar, "Colorization of Gray Scale Images in laß Color Space using Mean and Standard Deviation" IEEESCEECS 2012, MANIT, Bhopal (India), IEEE Conference Publications, March 2012.

[4] Welsh, T., Ashikhmin, M. and Mueller, K., "Transferring color to grey scale images", In: Proceedings of 29th Annual Conferenceon Computer Graphics and Interactive Techniques, ACM-SIGGRAPH2002, pp. 277- 280, ACM Press, New York, USA, 2002.

[5] DeepeshRawat and VijayaBhandari 'A Steganography Technique for Hiding Image in an Image using LSB Method for 24 Bit Color Image',International Journal of Computer Applications (0975 - 8887) Volume 64- No.20, February 2013

[6] GurmeetKaur and AartiKochhar 'A Steganography Implementation based on LSB \& DCT' International Journal for Science and Emerging ISSN No. (Online):2250-3641 Technologies with Latest Trends” 4(1): 35-41 (2012) 